\title{
BMJ Open Role of masks, testing and contact tracing in preventing COVID-19 resurgences: a case study from New South Wales, Australia
}

\author{
Robyn M Stuart (D) , ${ }^{1,2}$ Romesh G Abeysuriya, ${ }^{2}$ Cliff C Kerr, ${ }^{3,4}$ Dina Mistry, ${ }^{3}$ \\ Dan J Klein, ${ }^{3}$ Richard T Gray, ${ }^{5}$ Margaret Hellard, ${ }^{2,6,7,8}$ Nick Scott ${ }^{2}$
}

To cite: Stuart RM, Abeysuriya RG, Kerr CC, et al. Role of masks, testing and contact tracing in preventing COVID-19 resurgences: a case study from New South Wales, Australia. BMJ Open 2021;11:e045941. doi:10.1136/ bmjopen-2020-045941

- Prepublication history and additional materials for this paper is available online. To view these files, please visit the journal online (http://dx.doi. org/10.1136/bmjopen-2020045941).

Received 17 October 2020 Revised 16 March 2021 Accepted 19 March 2021
Check for updates

(C) Author(s) (or their employer(s)) 2021. Re-use permitted under CC BY-NC. No commercial re-use. See rights and permissions. Published by BMJ.

For numbered affiliations see end of article.

Correspondence to Dr Robyn M Stuart; robyn@math.ku.dk

\section{ABSTRACT}

Objectives The early stages of the COVID-19 pandemic illustrated that SARS-CoV-2, the virus that causes the disease, has the potential to spread exponentially. Therefore, as long as a substantial proportion of the population remains susceptible to infection, the potential for new epidemic waves persists even in settings with low numbers of active COVID-19 infections, unless sufficient countermeasures are in place. We aim to quantify vulnerability to resurgences in COVID-19 transmission under variations in the levels of testing, tracing and mask usage.

Setting The Australian state of New South Wales (NSW), a setting with prolonged low transmission, high mobility, non-universal mask usage and a well-functioning testand-trace system.

Participants None (simulation study).

Results We find that the relative impact of masks is greatest when testing and tracing rates are lower and vice versa. Scenarios with very high testing rates $(90 \%$ of people with symptoms, plus $90 \%$ of people with a known history of contact with a confirmed case) were estimated to lead to a robustly controlled epidemic. However, across comparable levels of mask uptake and contact tracing, the number of infections over this period was projected to be $2-3$ times higher if the testing rate was $80 \%$ instead of $90 \%, 8-12$ times higher if the testing rate was $65 \%$ or 30-50 times higher with a $50 \%$ testing rate. In reality, NSW diagnosed 254 locally acquired cases over this period, an outcome that had a moderate probability in the model $(10 \%-18 \%)$ assuming low mask uptake $(0 \%-25 \%)$, even in the presence of extremely high testing $(90 \%)$ and near-perfect community contact tracing $(75 \%-100 \%)$, and a considerably higher probability if testing or tracing were at lower levels.

Conclusions Our work suggests that testing, tracing and masks can all be effective means of controlling transmission. A multifaceted strategy that combines all three, alongside continued hygiene and distancing protocols, is likely to be the most robust means of controlling transmission of SARS-CoV-2.

\section{INTRODUCTION}

Across the world, governmental responses to the outbreak of the COVID-19 pandemic in
Strengths and limitations of this study

- A key methodological strength of this study is the level of detail in the model that we use, which allows us to capture many of the finer details of the extent to which controlling COVID-19 transmission relies on the balance between testing, contact tracing and mask usage.

- Another key strength is that our model is stochastic, so we are able to estimate the probability of different epidemiological outcomes under different policy settings.

- A key limitation is the shortage of publicly available data on the efficacy of contact tracing programmes, including data on how many people were contacted for each confirmed index case of COVID-19.

the first half of 2020 profoundly curtailed the spread of SARS-CoV-2, the virus that causes the disease. ${ }^{1-4}$ By mid-way through the year, an increasing number of countries had moved from an initial crisis management phase into a new phase centred around minimising transmission risk while allowing societal and economic activities to resume. ${ }^{5}$ However, by the end of 2020, many countries around the world had experienced epidemic resurgences necessitating further shutdowns. ${ }^{6}$ This was true even in settings that had come close to eliminating the virus, such as Vietnam, ${ }^{7} \mathrm{New}$ Zealand $^{8}$ and Australia. ${ }^{9}$ In low or zero transmission contexts, new outbreaks can emerge if community transmission has not been eliminated, or if infected people arrive from abroad or interstate and interact with the local community. ${ }^{10}$ It is therefore essential to be able to quantify the risk of epidemic resurgence under different policy contexts.

Given the complexities of COVID-19 transmission, including the duration of presymptomatic infection, ${ }^{11}$ 12 the proportion of infections that are asymptomatic ${ }^{13}$ and the 
possibility of transmission via surface contact, ${ }^{14}$ maintaining control of COVID-19 has proven challenging in many jurisdictions. ${ }^{15-19}$ The often-cited success stories of Taiwan, Vietnam, Thailand and South Korea included high mask usage, high rates of testing and fast and effective contact tracing. ${ }^{20-22}$ The benefits of a multipronged approach have also been illustrated in the literature; in the UK, for example, a recent study ${ }^{23}$ found that mandating masks in secondary schools could achieve approximately the same reduction in resurgence risk as having an $8 \%-11 \%$ increase in symptomatic testing.

In this work, we use an agent-based model to estimate the combination of testing, community-based contact tracing and mask usage required to maintain epidemic control in a low-transmission, high-mobility setting. These three non-pharmaceutical interventions (NPIs) were key components in reducing the probability of epidemic resurgences prior to the availability of a vaccine, and are likely to remain so for some time even after vaccination coverage increases. ${ }^{24}$ When used in combination with physical distancing and hand-washing/hygiene measures, these three strategies together can allow relatively high mobility: testing and contact tracing mean that only those at greatest risk of transmitting the virus need to stay home and have been shown to be effective in numerous settings, ${ }^{1525-29}$ while masks mean that people with undiagnosed infections present less of a risk to others. ${ }^{30-34}$

The context for our study is the Australian state of New South Wales (NSW), with a population of 7.5 million and a cumulative total of just over 4700 diagnosed cases as of 31 December 2020, of which the majority ( 57\%) were acquired overseas. After an initial wave of COVID-19 infections in March 2020 and subsequent lockdown in April, NSW began relaxing physical lockdown measures over May and was experiencing near-zero case counts by the start of June, with students back to school, businesses reopening and social/community activities resuming. In late June, several clusters of new infections were detected, which subsequently led to a 4-month-long period of low but steady case counts, with a mean of $\sim 5$ cases per day over July to October (excluding cases in quarantined travellers). However, NSW subsequently went on to record $\sim 180$ cases in the last 2 weeks of 2020 , a result of a localised outbreak whose containment necessitated the introduction of stringent new restrictions on travel and gatherings that affected many over the holiday period. ${ }^{35}$

During the prolonged period of low transmission that NSW experienced in the second half of 2020 prior to 15 December 2020, mobility remained high $^{36}$ and transmission was controlled via NPIs. Masks were recommended by the government for the general public and made mandatory for staff in various businesses including supermarkets, but were not universally adopted. In a survey undertaken in mid-September, $78 \%$ of the NSW population reported wearing a mask at some point in the previous week, ${ }^{37}$ although CCTV footage from August registered $\sim 30 \%$ of passengers on urban public transport wearing masks. ${ }^{38}$ At the same time, high levels of testing were in place, with $\sim 20000$ people tested per day over June to September ( 2.7/day per 1000 people), resulting in an average testing yield of $0.05 \%$, one of the world's lowest. ${ }^{39}$ The state also had a strong focus on contact tracing, with all cases interviewed within 1 day of case notification. ${ }^{40}$ Over the 4 months from 1 June to 30 September 2020, $\sim 900$ new cases were identified, but only $45(5 \%)$ were classified as 'source unknown', meaning that they were neither acquired overseas/interstate nor linked to known clusters. ${ }^{41}$

Relative to many other contact tracing programmes across the world, the NSW contact tracing programme was differentiated by its extensive efforts to identify a person's community contacts in addition to their household, social, school and workplace contacts. ${ }^{42}$ The state's health department (NSW Health) required all businesses to have a COVID-19 Safety Plan, and for the majority of public-facing businesses this included a requirement for customers to register their details on entry. On identifying a new case, NSW Health's contact tracers would then (A) conduct an extended interview to determine all possible venues in which transmission may have occurred, (B) place details of those venues on their website, on social media and in newspapers, urging people who had been at the venue to self-isolate for 14 days, and (C) attempt to contact all people who were registered to have been at venues within a given window of the time that the diagnosed case was known to have been there and instruct them to self-isolate for 14 days. ${ }^{42}$

The dynamics of COVID-19 transmission are complex, and in low-transmission settings the probability of maintaining epidemic control depends on numerous factors outside of policy control, including the characteristics of people who get infected: the size of their households, the type of work that they do and a number of other socioeconomic factors that may influence their contact networks. Several studies have pointed to the role of superspreading events and overdispersion of infections in COVID-19 transmission. ${ }^{25} 264344$ As a result, even with physical distancing, high levels of testing and rapid contact tracing, there is still a non-zero probability that a sustained outbreak could occur depending on who gets infected and where. In this study, we consider a range of testing and contact tracing levels, and assess the interacting roles of masks, testing and contact tracing as a means of controlling communitybased transmission.

\section{METHODS}

\section{Transmission model}

We used an open-source, agent-based model, Covasim, ${ }^{45}$ developed by the Institute for Disease Modeling with source code and documentation available at https:// covasim.org, and previously adapted by our group to model the Victorian epidemic. ${ }^{10}{ }^{46}$ Covasim contains detailed descriptions of age-dependent disease acquisition and progression probabilities and the duration of disease by acuity (see tables 1 and 2 in ref 45), as well 
as the effects of interventions including symptomatic and asymptomatic testing, isolation, contact tracing and quarantine, as well as other NPIs such as physical distancing, hygiene measures and protective equipment such as masks. Importantly, it also captures individual variability, with viral loads varying both between individuals and over time.

We began by simulating a population representative of NSW by taking data on the age and sex composition of the population from the 2016 census (the latest available), and using it to create a model population of agents with similar characteristics. The simulations consist of 100000 individual agents, who are dynamically scaled based on prevalence to represent the total NSW population of 7.5 million. The dynamical scaling means that whenever the proportion of susceptible agents falls below a threshold of $5 \%$, the number of agents in the model is increased; further implementation details can be found in Section 2.6.2 of Kerr et al. ${ }^{45}$

Next, we created contact networks for these agents. The governmental response to COVID-19 in NSW consisted of a set of highly context-specific policies covering individuals, businesses, schools and other types of organisations. To model these policies, we allow agents in the model to interact over five types of contact network: households, schools, workplaces and static and dynamic community networks. The static community network consists of interactions with friends, colleagues or other known associates who come together on a regular and predictable basis, and contains four subnetworks: professional sports, community sports/fitness/leisure clubs, places of worship and socialising with friends. The dynamic community network consists of interactions in which people interact with strangers or random groups of people, and contains seven separate subnetworks, representing: (1) arts venues such as museums, galleries, theatres and cinemas, (2) large events such as concerts, festivals, sports games, (3) pubs and bars, (4) cafes and restaurants, (5) public parks and other outdoor settings, (6) public transport, and (7) all other community settings. The method for constructing these networks is described in our previous study of the Victorian epidemic ${ }^{46}$ and is based on the methodology of the SynthPops Python package. ${ }^{47}$

Finally, to simulate the epidemic and policy environment in NSW during 1 March to 30 September 2020, we include parameter changes that capture the testing, tracing, isolation, quarantine and lockdown policies that were enacted over this time. Figure 1 presents a summary of how contact networks and the relative risk of COVID-19 transmission in different settings changed

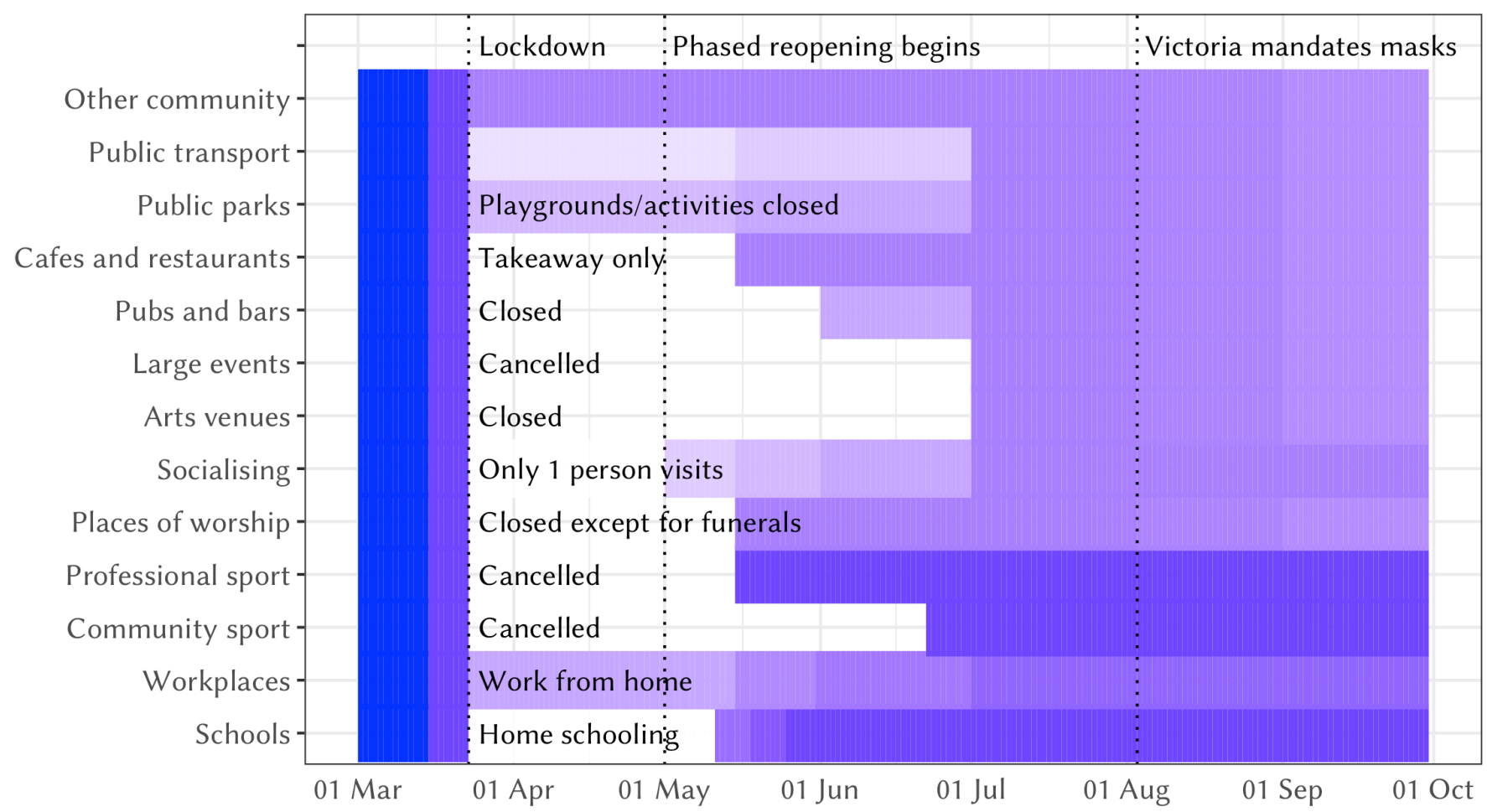

\section{Relative transmission risk}

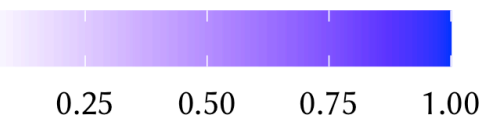

Figure 1 Relative changes in network structure and transmission risk across different settings in New South Wales over 1 March to 30 September, 2020. The absolute transmission risk varies by setting and is highest in household and lowest in outdoor settings (see ref 46 for details). 
as policies evolved. Some of these changes in transmission risk are derived from available data, ${ }^{48}$ while others are taken from a similar modelling exercise conducted in Victoria, in which a panel of Australia-based experts reviewed the likely effect of policies on transmission risks. ${ }^{46}$ Further details of all policies and how we model their effects on transmission risk are contained in online supplemental table S1. We also model the introduction of interstate cases in late June prior to the closure of the state border with neighbouring Victoria.

\section{Data and model fitting}

We calibrate the model by adjusting the per-contact transmission risk to minimise the absolute differences between the model projections and data on the daily number of cases diagnosed, excluding cases acquired overseas or interstate from NSW Health. ${ }^{41}$ Specifically, we drew 500 samples from a Gaussian prior distribution of values for the per-contact transmission risk $(\sim \mathrm{N}(0.025,0.002))$. For each sample, we run $\mathrm{N}=20$ simulations for each to produce 10000 trial simulations, and then retain the $1 \%$ of these with the minimum absolute differences between the model projections and the data.

\section{Model analysis and time frame}

As described above, we fit the model using data up until 30 September 2020. We then run the model for 3 months (from 1 October 2020 until 31 December 2020), under a set of assumptions about future testing rates, the efficacy of contact tracing and mask uptake. For each scenario (described below), we quantify the probability of the epidemic exceeding certain thresholds. We then compare these projections to the observed epidemic outcomes over the period from 1 October to 31 December 2020.

For testing rates, we distinguish between symptom-based testing and testing of asymptomatic contacts. NSW Health guidelines advise anyone with symptoms to get tested, as well as anyone identified as a contact regardless of symptoms. ${ }^{42}$ To reflect this, we use a baseline assumption that the testing rate for asymptomatic contacts is the same as for people with symptoms, and run a counterfactual set of scenarios in which it is assumed to be half the rate for those with symptoms (table 1). We modelled scenarios in which $50 \%, 65 \%, 80 \%$ or $90 \%$ of those with symptoms get tested. Test results are assumed to be communicated within 24 hours. ${ }^{49}$

We also considered variations on mask uptake and effectiveness. On uptake, we modelled scenarios in which $0 \%, 25 \%, 50 \%$ or $75 \%$ of the population will wear masks in dynamic community settings (ie, settings in which people interact with strangers or random groups of people), which in the model include arts venues, large events such as concerts, festivals, sports games, public parks and other outdoor settings, public transport and all other community settings. We do not consider scenarios in which $100 \%$ of the population wear masks across these settings, due to the infeasibility of wearing masks while eating or drinking. On efficacy, we note that although the
Table 1 Overview of the scenarios analysed during 1 October to 31 December 2020

\begin{tabular}{|c|c|}
\hline \multicolumn{2}{|l|}{ Core scenarios } \\
\hline $\begin{array}{l}4 \times \text { combinations of } \\
\text { symptomatic testing }\end{array}$ & $\begin{array}{l}50 \%, 65 \%, 80 \%-90 \% \text { tested } \\
\text { over the course of their } \\
\text { symptoms }\end{array}$ \\
\hline $\begin{array}{l}\text { Asymptomatic testing of } \\
\text { known contacts }\end{array}$ & $\begin{array}{l}\text { Asymptomatic contact testing } \\
\text { rate equal to symptomatic } \\
\text { testing rate }\end{array}$ \\
\hline $\begin{array}{l}5 \times \text { combinations of } \\
\text { contact tracing for } \\
\text { community contacts }\end{array}$ & $\begin{array}{l}0 \%, 25 \%, 50 \%, 100 \% \text { of } \\
\text { contacts traced within } 1 \text { week } \\
\text { (Poisson distribution with a } \\
\text { mean of } 1 \text { day) }\end{array}$ \\
\hline $\begin{array}{l}4 \times \text { combinations of } \\
\text { mask uptake }\end{array}$ & $0 \%, 25 \%, 50 \%, 75 \%$ \\
\hline Mask efficacy & $30 \%$ \\
\hline \multicolumn{2}{|l|}{ Sensitivity analyses } \\
\hline $\begin{array}{l}\text { Sensitivity to } \\
\text { asymptomatic contact } \\
\text { testing rate assumption }\end{array}$ & $\begin{array}{l}\text { We run the same } 80(4 \times 5 \times 4) \\
\text { scenarios described above, } \\
\text { but with the asymptomatic } \\
\text { contact testing rate half of the } \\
\text { symptomatic testing rate. }\end{array}$ \\
\hline $\begin{array}{l}\text { Sensitivity to the mask } \\
\text { efficacy assumption }\end{array}$ & $\begin{array}{l}\text { We run the same } 80(4 \times 5 \times 4) \\
\text { scenarios described above, but } \\
\text { with individual mask efficacy } \\
\text { assumed to be } 15 \% \text { or } 45 \% \\
\text { instead of } 30 \% \text {. }\end{array}$ \\
\hline
\end{tabular}

body of evidence supporting the effectiveness of masks for protecting against transmission between individuals is now considerable, the size of the effect is difficult to determine, with estimates in the range of $20 \%-80 \%$ and varying depending on whether one or both parties wear masks, ${ }^{31} 5051$ or whether spillover behavioural changes on people's attention to other NPIs are captured. ${ }^{52}$ To capture the uncertainty regarding the effectiveness of masks, we assume that masks will reduce the per-contact probability of transmission by $30 \%$, in line with estimates from ref 53 but also consider $15 \%$ and $45 \%$ in a sensitivity analysis presented in the online supplemental materials. We do not model the differences between both people wearing masks versus source only versus target only, so the estimates we use here should be considered as averages across these different possibilities.

To model the efficacy of contact tracing, we use publicly available data from NSW Health. ${ }^{42}$ We note that, although the programme reports the proportion of known contacts that were reached within defined time frames, we would ideally like to know the proportion of all contacts that were reached, which will be lower than the reported values since it will also include contacts that the case did not recall or disclose. Thus, although NSW Health's published reports ${ }^{42}$ indicate that $100 \%$ of contacts are notified within 48 hours, we use slightly more conservative values, namely that $100 \%$ of household contacts will be traced and notified on the same day that test results are 
communicated, and that $95 \%$ of school contacts and $90 \%$ of workplace contacts will be notified on the following day. We then consider scenarios in which $0 \%, 25 \%, 50 \%$, $75 \%$ or $100 \%$ of all other contacts (which we refer to as community contacts) can be traced within a week of a case notification. For each scenario, the time to trace each contact is drawn from a scaled Poisson distribution with a mean of 1 day (online supplemental figure $\mathrm{S} 1$ ). We run 100 simulations for each permutation of testing rates, mask usage and contact tracing efficacy.

Finally, we assume that people who have been contact traced will quarantine with $90 \%$ compliance from their workplace, school and community contacts. While this assumption may be optimistic in other global contexts, the lower case counts in NSW mean that contact tracers have far greater capacity for rigorous ongoing follow-up of contacts, and breaches of isolation are escalated with local authorities, as stipulated in national guideline documents. ${ }^{50}$

\section{Role of the funding source}

The funders had no role in the design or execution of this study.

\section{Patient and public involvement}

This is a modelling study; no personal data were used and patient/public involvement was not required.

\section{RESULTS}

According to our modelled estimates, $4550 \quad(95 \%$ projected interval: 3700-6660) people had acquired COVID-19 in NSW by the end of September 2020 (excluding cases acquired overseas or interstate), of which $39 \%(27 \%-48 \%)$ had been diagnosed (figure 2). The majority of undiagnosed infections occurred during the March to April wave; since the beginning of June, we estimate that $65 \%$ of all locally acquired infections have been diagnosed, with the remaining $35 \%$ primarily comprising asymptomatic infections $(65 \%)$. We further estimate that 79330 (60 100-129 020) people had been required to self-isolate at some point by 30 September 2020 as a result of having potentially been in contact with a confirmed case.

Estimates of daily new infections under each combination of testing, community contact tracing and mask usage are presented in figure 3. A key finding highlighted by figure 3 is how effective high levels of testing are in maintaining epidemic control: all strategies in which there is at least some contact tracing in place and testing rates are very high $(90 \%$ of people with symptoms and $90 \%$ of asymptomatic contacts of confirmed cases) lead to a robustly controlled epidemic, with a median of $\sim 180$ infections in total estimated during 1 October to 31 December 2020 under high mask uptake scenarios, or 260-1200 without masks, depending on the efficacy of community contact tracing (figure 3, bottom row). However, holding mask uptake and contact tracing constant, we estimate that the number of infections during 1 October to 31 December 2020 would be 2-3 times higher if the testing rate was $80 \%$ instead of $90 \%, 8-12$ times higher if the testing rate was $65 \%$ or $30-50$ times higher with a $50 \%$ testing rate (figure 3 , third row).

A second key finding is that the lower the testing rate, the greater the impact of masks and contact tracing (and vice versa). With medium-lower testing rates of $50 \%$ or $65 \%$, the marginal impact of both masks and contact tracing is considerable (figure 3, top two rows). Under these scenarios, the most robust strategies consist of a combination of masks and community contact tracing. Assuming a $50 \%$ testing rate, a scenario with near-perfect tracing and no masks is approximately equivalent to a scenario with no tracing and high mask uptake. However, without community contact tracing, a reduction in mask usage from $75 \%$ to $50 \%$ would lead to a near doubling in estimated infections (from 7900 to 17000 ), whereas even moderate levels of community contact tracing (25\% traceable within a week) would increase the robustness of the response to lower mask usage (with 6400 infections
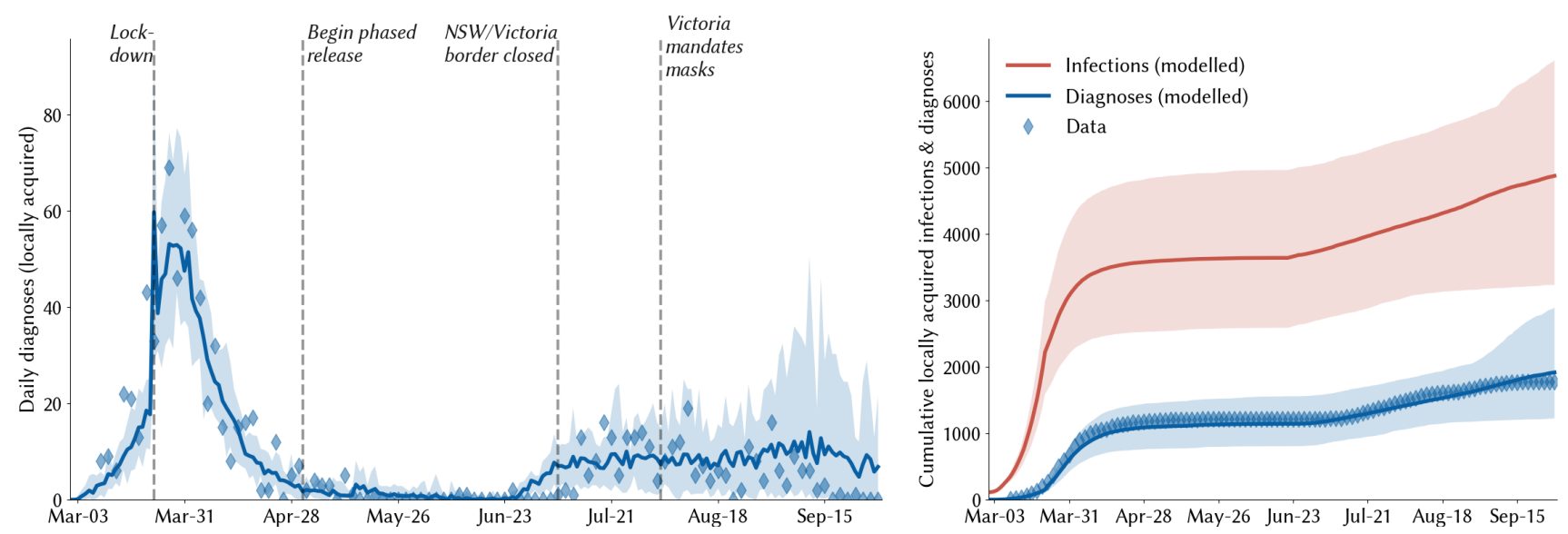

Figure 2 Calibration of the model to the New South Wales (NSW) epidemic. Solid lines indicate the median model projections over 100 model runs; shaded areas indicate 95\% projected intervals over different initialisations; blue diamonds indicate data on confirmed locally acquired cases. 

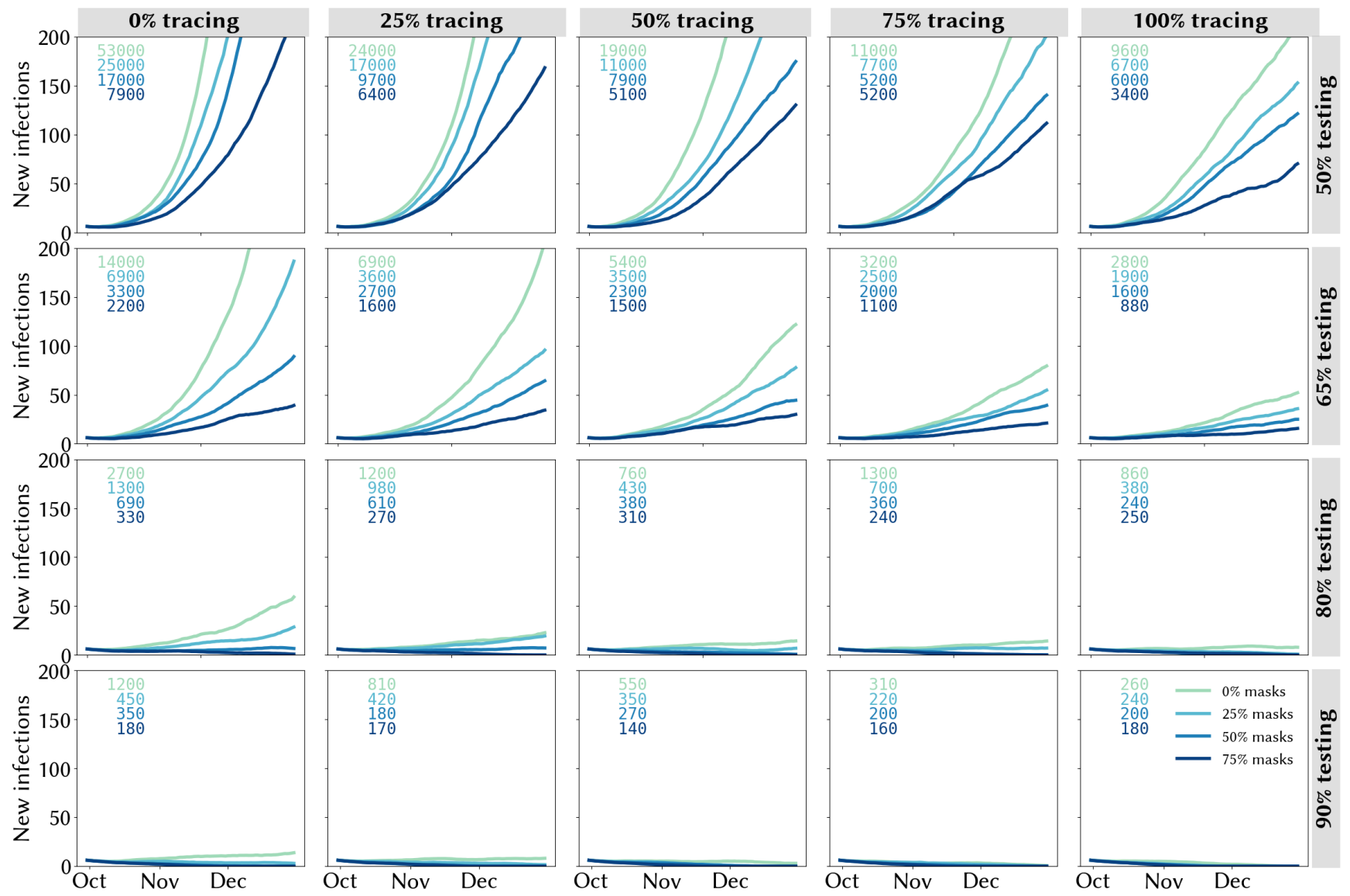

Figure 3 Trailing 14-day average of daily locally acquired infections under different assumptions about the testing rate (rows), proportion of community contacts that can be traced within 1 week (columns) and mask uptake (line colours). Projections represent the median of 100 simulations. Text boxes in each panel display the cumulative number of infections during 1 October to 31 December 2020.

estimated under $75 \%$ mask usage and 9700 under $50 \%$ mask usage). Similarly, masks strengthen the resilience of the epidemic outcome to decreases in contact tracing efficacy: without masks, a reduction in community contact tracing from $75 \%$ to $50 \%$ would lead to a $70 \%$ increase in total infections, whereas it would have no impact if $75 \%$ of the population were wearing masks.

Figure 4 captures the risks associated with different strategies, quantified in terms of the probability of the 14-day average of daily diagnoses exceeding a given number by 31 December 2020. With no community tracing and a $50 \%$ testing rate, the probability of exceeding 100 diagnoses/day by the end of 2020 was calculated to be $98 \%$ without masks, and to remain greater than $50 \%$ even under the most optimistic mask uptake scenario (figure 4, top left panel). However, higher levels of community tracing reduce these risks considerably: even without any mask usage, the probability of exceeding 100 cases/day is estimated to be half as high with perfect community tracing (figure 4, top right panel) compared with the same scenario without community tracing ( $48 \%$ vs $98 \%$ ), and the addition of mask usage in community settings is estimated to reduce this even further to $\sim 10 \%$.

\section{Comparison with actual outcomes}

The text boxes in figure 4 display the probability of observing the outcome that was seen in NSW, in which the 2-week average of daily diagnoses exceeded 12 by 31 December. We calculate that this outcome would have been assigned a low probability $(4 \%-7 \%)$ under the best-case scenarios of extremely high testing (90\%), nearperfect community contact tracing $(75 \%-100 \%)$ and high mask usage $(50 \%-75 \%)$, but that it would have been considered a far more likely outcome if any of these were assumed to be at lower levels.

\section{Sensitivity analyses}

If asymptomatic contacts only test at half the rate of people with symptoms, we estimate a more severe epidemic over the last quarter of 2020, but the key results regarding the roles of community contact tracing and masks do not change (online supplemental figure S2). Since our core analyses already assume high compliance with recommended self-isolation policies for known contacts of confirmed cases, the marginal benefit of high asymptomatic contact tracing is primarily to further bolster the efficacy of contact tracing, since it allows for 

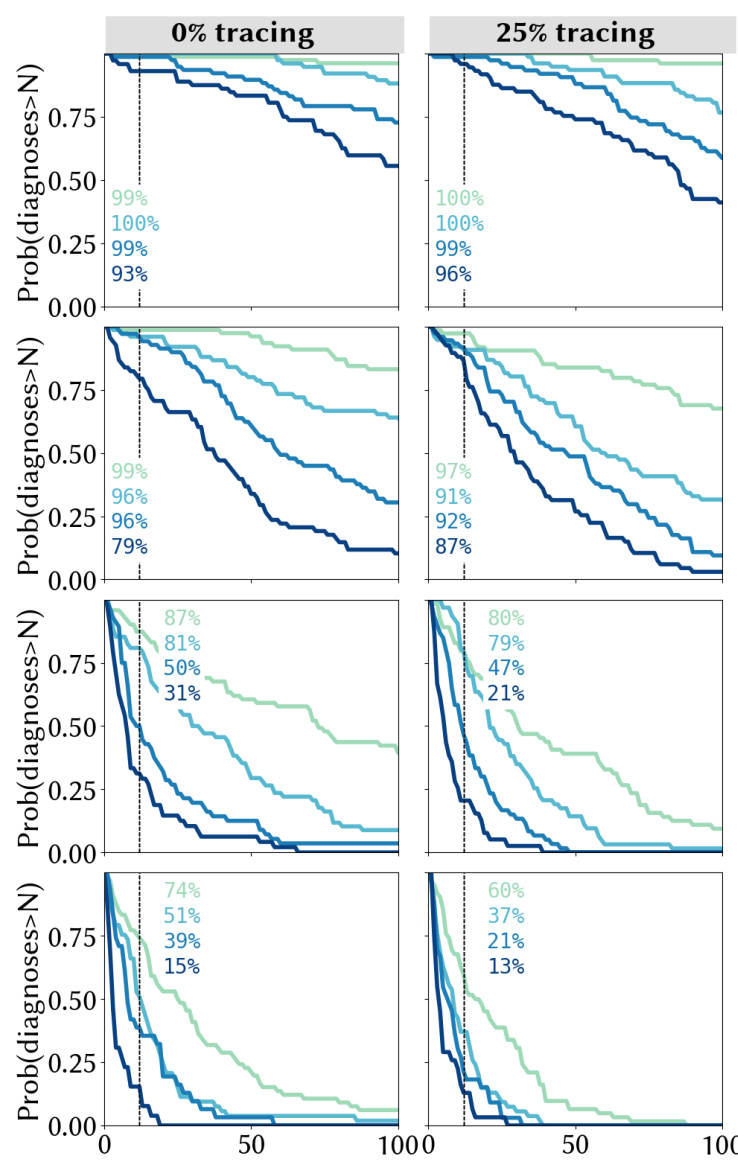
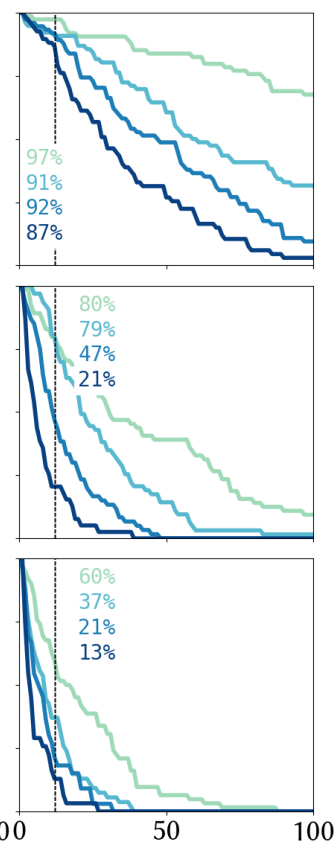
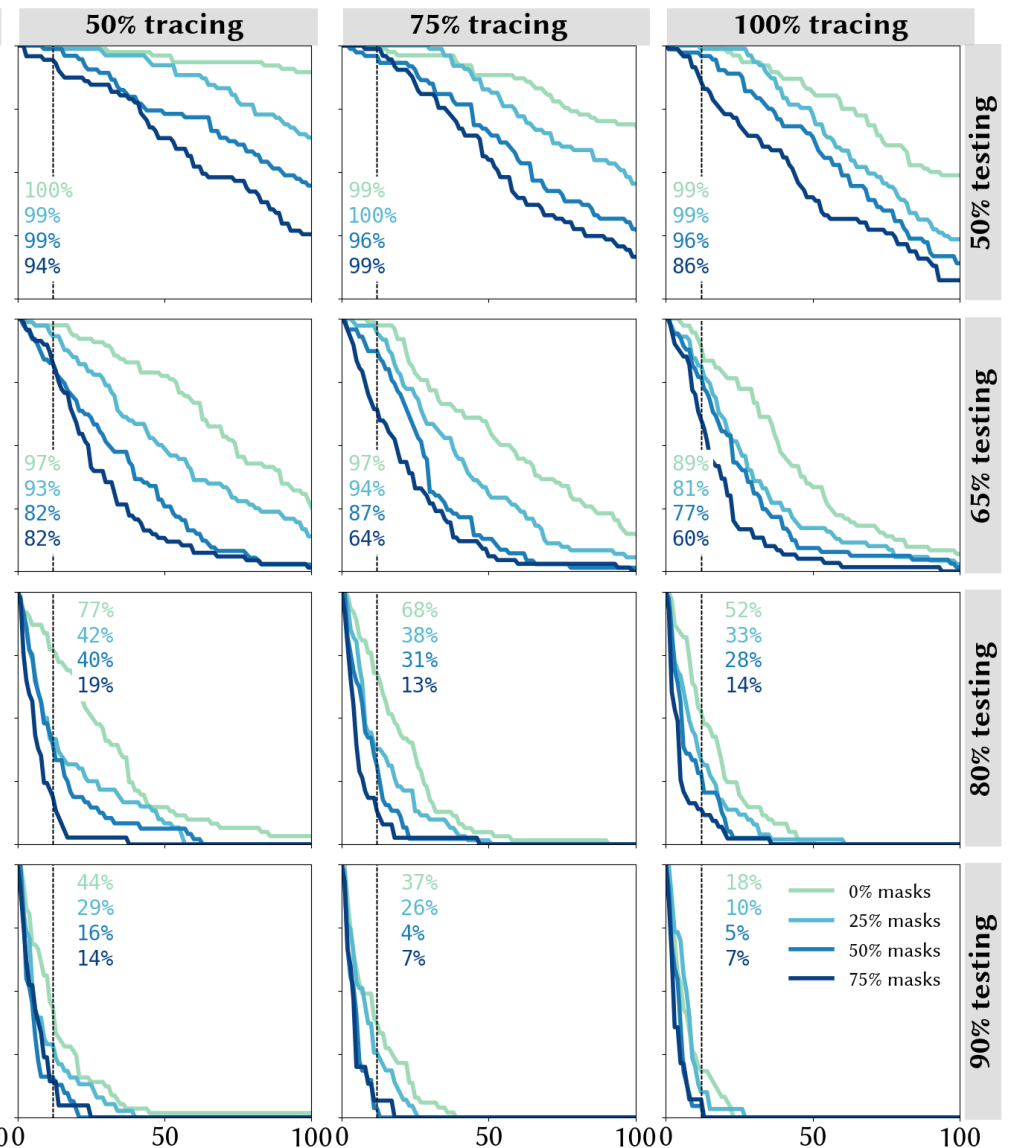

Figure 4 Quantifying the likelihood of epidemic resurgence in New South Wales: the probability of the trailing 14-day average of locally acquired cases exceeding a given number by 31 December 2020 under different assumptions about the testing rate (rows), proportion of community contacts that can be traced within 1 week (columns) and mask uptake (line colours).

the identification of chains of transmission even in the absence of symptoms. To highlight the role of testing asymptomatic contacts, in figure 5 we highlight a particular set of scenarios corresponding to the most optimistic contact tracing assumptions, in which all community contacts are assumed to be traced within 1 week, with a mean time to trace of 1 day (the full set of scenarios is summarised in online supplemental figure S2). Within this set of scenarios, the total number of infections is estimated to be around $50 \%$ higher if asymptomatic contacts test at a lower rate than people with symptoms (averaged across all levels of mask usage).
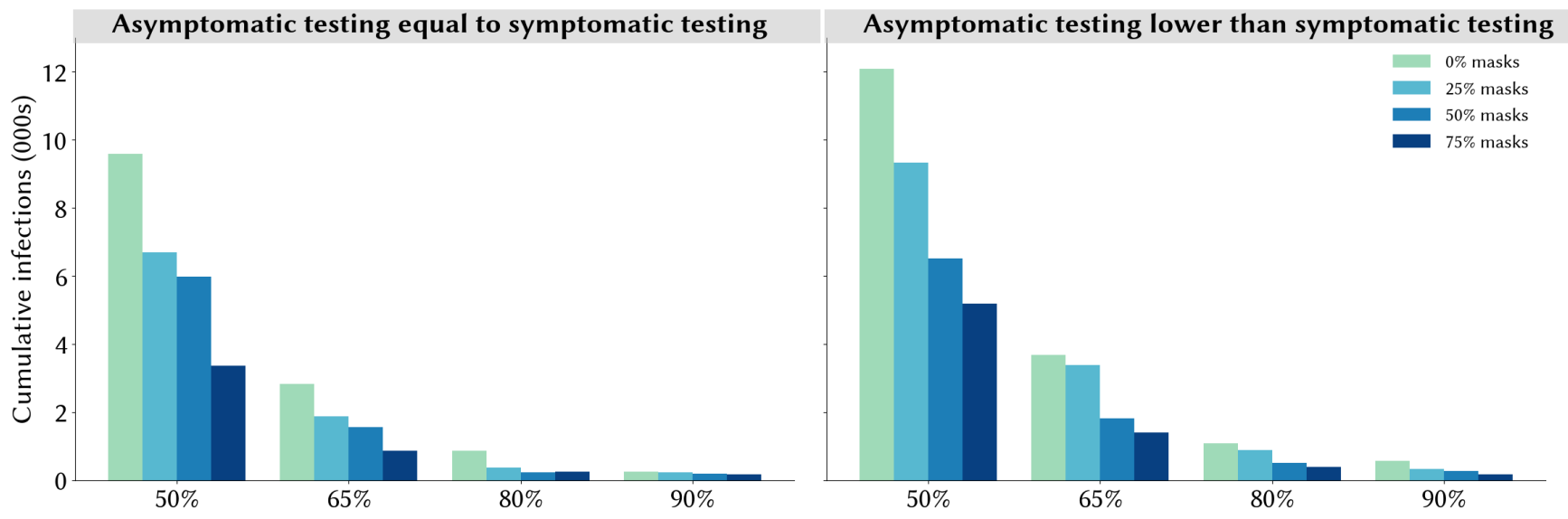

Figure 5 Estimated total infections during1 October to 31 December 2020 under different assumptions about testing rates and mask uptake, assuming all community contacts can be traced within a week with a mean time to trace of 1 day. Projections represent the median of 100 simulations. 
We also found that, if masks are assumed to be more or less effective, this would change the risks associated with diagnosing more than a given number of cases per day under the different mask scenarios, but would not qualitatively change the results (online supplemental figures S3 and S4). For example, we estimate 1500 infections over the 3-month period assuming 50\% tracing, 65\% symptomatic testing and $75 \%$ mask uptake if masks have an efficacy of $30 \%$ (figure 3, central panel), compared with 1800 infections if masks had $15 \%$ efficacy or 1300 infections if masks had $45 \%$ efficacy.

\section{DISCUSSION}

In this work we presented estimates illustrating the extent to which masks, community testing and community contact tracing could reduce the spread of SARS-CoV-2 in NSW, Australia, in the absence of widespread vaccination. We found that the relative impact of masks is greatest when testing and tracing rates are lower (and vice versa). With very high testing rates $(90 \%$ of people with symptoms, plus $90 \%$ of people with a known history of contact with a confirmed case), we estimate that epidemic control would be possible even without widespread mask uptake, provided that fast and effective contact tracing is in place. However, under any scenario where testing rates are lower, we estimate that mask use can play an important role in reducing the potential for epidemic resurgence.

Our findings have relevance for assessing the outbreak that occurred in the state towards the end of December 2020 , which saw an average of $\sim 12$ diagnoses/day over 2 weeks. According to our estimates, this outcome would have been assigned a relatively low probability $(4 \%-7 \%)$ if it was assumed that compliance with the state's testing guidelines, venue registration protocols and nonmandatory mask recommendations would be at very high levels, but that it would have been evaluated as a far more likely outcome if compliance with any of these NPIs was assumed to be lower. As a result of the December 2020 outbreak, the NSW government made masks mandatory in all indoor community settings from 4 January $2021,{ }^{54}$ a decision which will likely increase the robustness of the overall response to decreases in testing or contact tracing rates, but will still require ongoing commitment to ensure these remain at high levels, at least until vaccination coverage is sufficiently high. According to Australia's vaccination schedule, half of the population may be vaccinated by mid-2021, and NPIs will feature as part of the COVID-19 control strategy until well beyond this point. ${ }^{55}$

This study suggests that maintaining high levels of symptomatic testing, contact tracing and testing of contacts are all important. We quantified the importance of testing asymptomatic contacts as a strategy that further strengthens the power of contact tracing programmes, since it allows the tracing and quarantining of their contacts in turn. The interdependencies between different arms of testing and tracing strategies are complex, as testing of asymptomatic contacts is only feasible if contact tracing is effective enough to identify them. Longer term, 'pandemic fatigue' may bring about challenges in maintaining high levels of testing, mask use and contact tracing. ${ }^{56}$ This study suggests that having high levels of any two of mask use, testing and contact tracing can largely mitigate the need for the third; however, practical challenges mean that this is unlikely to occur, and a more multifaceted approach of aiming for high coverage of all three and ending up with moderate coverage of all three may be an effective and more robust strategy. Various studies have shown that the roll-out of vaccination plans is unlikely to mitigate the need for NPIs such as these for some time, ${ }^{24}{ }^{57-59}$ so these findings are likely to remain relevant for a considerable portion of 2021.

Although various efforts have been made to synthesise the ever-expanding body of research regarding the efficacy of different interventions, ${ }^{1-460}$ each country's epidemic has distinct characteristics and there are very few standardised, globally applicable guidelines on what constitutes a bestpractice public health strategy. ${ }^{61}{ }^{62}$ As a result, jurisdictions have taken diverse approaches in terms of which interventions to prioritise from a list that includes physical distancing, travel restrictions, wearing of masks or face coverings, isolation and/or testing of those with COVID-like symptoms, isolation of those who test positive and tracing the contacts of confirmed cases for testing and/or quarantining. Although it may not always be articulated as such, numerous trade-offs are being made between different policy options in an attempt to allow the highest degree of societal activity commensurate with epidemic control. In NSW, mask use was encouraged in particular settings since July 2020, but not mandated until January 2021; at the same time, there was a strong focus on contact tracing. The results from this work suggest that the prioritisation of contact tracing may mitigate the relative importance of masks to some extent, but that this relies on continued high levels of community testing.

There are several limitations to this study. First, the mathematical model that we use requires data on various aspects of SARS-CoV-2 transmission and prevention that are still not known exactly, including the effects of masks on preventing individual transmission and the proportion of infections that are asymptomatic. While we have used the best available data and sampled from appropriate distributions where possible, this represents a source of uncertainty in all mathematical models of COVID-19. Additional uncertainties are introduced by the evolution of new strains of SARS-CoV-2 with increased transmissibility and/or severity. Second, we have constructed sets of scenarios that examine various combinations of parameters on mask uptake, contact tracing, testing of people with symptoms and asymptomatic contact testing, but there are many more parameters that determine the dynamics of transmission, including the stringency of border control measures, people's adherence to quarantine and isolation policies and the effect of ongoing distancing policies. Changes to any of these policies would affect the results presented here in ways that are not straightforward to predict or extrapolate. Third, we have not considered any 
outbreak risk associated with newly seeded cases in the community that may arise from international or interstate arrivals. Another limitation is the relatively simplistic way that we have modelled mask usage, whereby we have not included variation in (A) adherence to mask usage across different types of venue, or (B) individual compliance. Assuming that mask wearing reduces everyone's transmission risk by a certain percentage disregards the individual behavioural changes that may adjust individual-level transmission risk by varying amounts. Furthermore, it is very likely that mask usage would be higher in certain settings (eg, public transport) than others, especially restaurants which are suspected to be important for transmission. Finally, the model used here does not contain a geospatial component, and we have not considered heterogeneities in incidence, behaviour or contact patterns across different parts of the state in these analyses. This could be relevant for questions around mask uptake, as uptake of masks is generally higher in more densely populated areas, so using a state-wide average for the proportion of the population wearing masks may underestimate their impact, especially since $>90 \%$ of infections in NSW to date have occurred in the Sydney metropolitan area.

\section{CONCLUSIONS}

Our work suggests that testing, tracing and masks can be effective means of controlling transmission in dynamic community settings, and higher compliance with one can offset lower compliance with the other to some extent. However, pursuing a strategy that combines aggressive testing, high mask usage and effective contact tracing, alongside continued hygiene and distancing protocols, is likely to be the most robust means of controlling community-based transmission of SARS-CoV-2.

\section{Author affiliations}

${ }^{1}$ Department of Mathematical Sciences, University of Copenhagen, Copenhagen, Denmark

${ }^{2}$ Burnet Institute, Melbourne, Victoria, Australia

${ }^{3}$ Global Health Division, Institute for Disease Modeling, Bill \& Melinda Gates

Foundation, Seattle, Washington, USA

${ }^{4}$ School of Physics, University of Sydney, Sydney, New South Wales, Australia

${ }^{5}$ The Kirby Institute, UNSW Sydney, Sydney, New South Wales, Australia

${ }^{6}$ Department of Infectious Diseases, The Alfred Hospital, Melbourne, Victoria, Australia

${ }^{7}$ Department of Epidemiology and Preventive Medicine, Monash University, Melbourne, Victoria, Australia

${ }^{8}$ Doherty Institute and School of Population and Global Health, University of Melbourne, Melbourne, Victoria, Australia

Acknowledgements We thank all contributors to the Covasim model, including at the Institute for Disease Modeling, including Brittany Hagedorn, Katherine Rosenfeld, Prashanth Selvaraj, Rafael Nunez, Gregory Hart, Carrie Bennette, Marita Zimmermann, Assaf Oron, Dennis Chao, Michael Famulare and Lauren George; at Github, including Michał Jastrzębski, Will Fitzgerald, Cory Gwin, Julian Nadeau, Hamel Husain, Rasmus Wriedt Larsen, Aditya Sharad and Oege de Moor; at Microsoft, including William Chen, Scott Ayers and Rolf Harms; and at the Burnet Institute, including Anna Palmer, Dominic Delport and Sherrie Kelly. This work benefited from a useful discussion with James Wood, Deborah Cromer and John Kaldor of the University of NSW, Australia.
Contributors RMS wrote the manuscript and produced the results. The model parameters and calibration were agreed upon by RMS, RGA, NS and RTG. The scenarios and analyses were agreed upon by NS, MH and RMS. CCK, RMS, RGA, DM and DJK led the development of the model (along with numerous other contributors).

Funding The authors have not declared a specific grant for this research from any funding agency in the public, commercial or not-for-profit sectors.

Competing interests None declared.

Patient consent for publication Not required.

Provenance and peer review Not commissioned; externally peer reviewed.

Data availability statement Data are available in a public, open-access repository. All codes and data analysed during this study are available on https:// github.com/optimamodel/covid_nsw.

Supplemental material This content has been supplied by the author(s). It has not been vetted by BMJ Publishing Group Limited (BMJ) and may not have been peer-reviewed. Any opinions or recommendations discussed are solely those of the author(s) and are not endorsed by BMJ. BMJ disclaims all liability and responsibility arising from any reliance placed on the content. Where the content includes any translated material, BMJ does not warrant the accuracy and reliability of the translations (including but not limited to local regulations, clinical guidelines, terminology, drug names and drug dosages), and is not responsible for any error and/or omissions arising from translation and adaptation or otherwise.

Open access This is an open access article distributed in accordance with the Creative Commons Attribution Non Commercial (CC BY-NC 4.0) license, which permits others to distribute, remix, adapt, build upon this work non-commercially, and license their derivative works on different terms, provided the original work is properly cited, appropriate credit is given, any changes made indicated, and the use is non-commercial. See: http://creativecommons.org/licenses/by-nc/4.0/.

ORCID iD

Robyn M Stuart http://orcid.org/0000-0001-5482-9584

\section{REFERENCES}

1 Flaxman S, Mishra S, Gandy A, et al. Estimating the effects of non-pharmaceutical interventions on COVID-19 in Europe. Nature 2020;584:257-61.

2 Haug N, Geyrhofer L, Londei A, et al. Ranking the effectiveness of worldwide COVID-19 government interventions. Nat Hum Behav 2020;4:1303-12.

3 Dehning J, Zierenberg J, Spitzner FP, et al. Inferring change points in the spread of COVID-19 reveals the effectiveness of interventions. Science 2020;369:eabb9789.

4 Hsiang S, Allen D, Annan-Phan S, et al. The effect of large-scale anti-contagion policies on the COVID-19 pandemic. Nature 2020;584:262-7.

5 Han E, MMJ T, Turk E, et al. Lessons learnt from easing COVID-19 restrictions: an analysis of countries and regions in Asia Pacific and Europe. Lancet 2020 https://www.thelancet.com/journals/lancet/ article/PIIS0140-6736(20)32007-9/abstract

6 The Independent. Which countries are in lockdown? [online], 2020. Available: https://www.independent.co.uk/news/world/whichcountries-currently-in-lockdown-b1777806.html [Accessed 25 Jan 2021].

7 Nong VM, Le Thi Nguyen Q, Doan TT, et al. The second wave of COVID-19 in a tourist hotspot in Vietnam. $J$ Travel Med 2021;28.

8 the Guardian. "Here we go again": Auckland fears a long lockdown as coronavirus returns [online], 2020. Available: http://www. theguardian.com/world/2020/aug/13/here-we-go-again-aucklandfears-a-long-lockdown-as-coronavirus-returns [Accessed 25 Jan 2021].

9 BBC News. Coronavirus: Victoria declares state of disaster after spike in cases [online], 2020. Available: https://www.bbc.com/news/ world-australia-53627038 [Accessed 25 Jan 2021].

10 Abeysuriya R, Delport D, Stuart R, et al. Preventing a cluster from becoming a new wave in settings with zero community COVID-19 cases. doi:10.1101/2020.12.21.20248595

$11 \mathrm{He}$ X, Lau EHY, Wu P, et al. Temporal dynamics in viral shedding and transmissibility of COVID-19. Nat Med 2020;26:672-5.

12 Slifka MK, Gao L. Is presymptomatic spread a major contributor to COVID-19 transmission? Nat Med 2020;26:1531-3.

$13 \mathrm{He} \mathrm{J}$, Guo Y, Mao R. Proportion of asymptomatic coronavirus disease 2019: a systematic review and meta-analysis. J Med Virol 2020. 
14 Chin AWH, Chu JTS, Perera MRA, et al. Stability of SARS-CoV-2 in different environmental conditions. Lancet Microbe 2020;1:e10.

15 Hellewell J, Abbott S, Gimma A, et al. Feasibility of controlling COVID-19 outbreaks by isolation of cases and contacts. Lancet Glob Health 2020;8:e488-96.

16 Fraser C, Riley S, Anderson RM, et al. Factors that make an infectious disease outbreak controllable. Proc Natl Acad Sci U S A 2004;101:6146-51.

17 Keeling MJ, Hollingsworth TD, Read JM. Efficacy of contact tracing for the containment of the 2019 novel coronavirus (COVID-19). $\mathrm{J}$ Epidemiol Community Health 2020;74:861-6.

18 lacobucci G. Covid-19: is local contact tracing the answer? BMJ 2020;370:m3248.

19 Ferretti L, Ledda A, Wymant C, et al. The timing of COVID-19 transmission. medRxiv 2020. doi:10.2139/ssrn.3716879

20 Wang CJ, Ng CY, Brook RH. Response to COVID-19 in Taiwan: big data analytics, new technology, and proactive testing. JAMA 2020;323:1341-2.

21 Duong DM, Le VT, Ha BTT. Controlling the COVID-19 pandemic in Vietnam: lessons from a limited resource country. Asia Pac J Public Health 2020;32:161-2.

22 You J. Lessons from South Korea's covid-19 policy response. Am Rev Public Admin 2020;50:801-8.

23 Panovska-Griffiths J, Kerr CC, Waites W. The potential contribution of face coverings to the control of SARS-CoV-2 transmission in schools and broader society in the UK: a modelling study. medRxiv 2020. doi:10.1101/2020.09.28.20202937

24 Conlen M, Lu D, Glanz J. Why vaccines alone will not end the pandemic. The New York Times [online], 2021. Available: https:// www.nytimes.com/interactive/2021/01/24/us/covid-vaccine-rollout. html [Accessed 25 Jan 2021].

25 Sun K, Wang W, Gao L, et al. Transmission heterogeneities, kinetics, and controllability of SARS-CoV-2. Science 2021;371 doi:10.1126/ science.abe2424

26 Kerr CC, Mistry D, Stuart RM. Controlling COVID-19 via test-tracequarantine [online] 2020. doi:10.1101/2020.07.15.20154765

27 Kretzschmar ME, Rozhnova G, Bootsma MCJ, et al. Impact of delays on effectiveness of contact tracing strategies for COVID-19: a modelling study. Lancet Public Health 2020;5:e452-9.

28 Kucharski AJ, Klepac P, Conlan AJK, et al. Effectiveness of isolation, testing, contact tracing, and physical distancing on reducing transmission of SARS-CoV-2 in different settings: a mathematical modelling study. Lancet Infect Dis 2020;20:1151-60. doi:10.1016/ S1473-3099(20)30457-6

29 Ferretti L, Wymant C, Kendall M, et al. Quantifying SARS-CoV-2 transmission suggests epidemic control with digital contact tracing. Science 2020;368 doi:10.1126/science.abb6936

30 Eikenberry SE, Mancuso M, Iboi E, et al. To mask or not to mask: modeling the potential for face mask use by the general public to curtail the COVID-19 pandemic. Infect Dis Model 2020;5:293-308.

31 Chu DK, Akl EA, Duda S, et al. Physical distancing, face masks, and eye protection to prevent person-to-person transmission of SARS-CoV-2 and COVID-19: a systematic review and meta-analysis. Lancet 2020;395:1973-87.

32 Catching A, Capponi S, Yeh MT. Examining face-mask usage as an effective strategy to control COVID-19 spread. Epidemiology 2020. doi:10.1101/2020.08.12.20173047

33 Javid B, Balaban NQ. Impact of population mask wearing on Covid-19 post lockdown. Infect Micro Dis 2020.

34 Mitze T, Kosfeld R, Rode J, et al. Face masks considerably reduce COVID-19 cases in Germany. PNAS [online], 2020. Available: https:// www.pnas.org/content/early/2020/12/02/2015954117 [Accessed 4 Dec 2020].

35 Covid in Sydney: New restrictions announced as outbreak grows - BBC News [online]. Available: https://www.bbc.com/news/worldaustralia-55378180 [Accessed 25 Jan 2021].

36 COVID-19 community mobility reports [online]. Available: https:// www.google.com/covid19/mobility/ [Accessed 25 Jan 2021].

37 Australian Bureau of Statistics. Household impacts of COVID-19 survey, August 2020 [online]. Available: https://www.abs.gov.au/ statistics/people/people-and-communities/household-impactscovid-19-survey/latest-release [Accessed 28 Sep 2020].

38 The Sydney Morning Herald. CCTV monitors how many are wearing masks on trains, 2020. Available: https://www.smh.com.au/national/ nsw/cctv-monitors-how-many-are-wearing-masks-on-trains20200813-p55lh4.html [Accessed 2 Sep 2020].

39 Roser M, Ritchie H, Ortiz-Ospina E, et al. Coronavirus pandemic (COVID-19). Our world in data [online], 2020. Available: https:// ourworldindata.org/coronavirus [Accessed 25 Jan 2021].
40 COVID-19 weekly surveillance reports - COVID-19 (Coronavirus) [online]. Available: https://www.health.nsw.gov.au/Infectious/covid19/Pages/weekly-reports.aspx [Accessed 25 Aug 2020]

41 NSW COVID-19 data | Data NSW [online]. Available: https://data.nsw. gov.au/nsw-covid-19-data [Accessed 28 Sep 2020].

42 COVID-19: Updated advice on testing, 24 September 2020 COVID-19 (Coronavirus) [online]. Available: https://www.health.nsw. gov.au/Infectious/covid-19/Pages/case-definition.aspx [Accessed 28 Sep 2020].

43 Endo A, Abbott S, et al, Centre for the Mathematical Modelling of Infectious Diseases COVID-19 Working Group. Estimating the overdispersion in COVID-19 transmission using outbreak sizes outside China. Wellcome Open Res 2020;5:67.

44 Althouse BM, Wenger EA, Miller JC, et al. Stochasticity and heterogeneity in the transmission dynamics of SARS-CoV-2. arXiv:200513689 [physics, q-bio] [online], 2020. Available: https:// journals. plos.org/plosbiology/article?id=10.1371/journal.pbio. 3000897 [Accessed 29 Aug 2020].

45 Kerr CC, Stuart RM, Mistry D, et al. Covasim: an agent-based model of COVID-19 dynamics and interventions, 2020. Available: https://www.medrxiv.org/content/10.1101/2020.05.10.20097469v1 [Accessed 19 Aug 2020].

46 Scott N, Palmer A, Delport D, et al. Modelling the impact of reducing control measures on the COVID-19 pandemic in a low transmission setting, 2020. Available: https://www.mja.com.au/journal/2020/ modelling-impact-reducing-control-measures-covid-19-pandemiclow-transmission-setting [Accessed 19 Aug 2020].

47 Institute for Disease Modeling. Institute for disease modeling/ synthpops [online]. Available: https://github.com/InstituteforDise aseModeling/synthpops [Accessed 21 Aug 2020].

48 ABC News. Recovering from coronavirus: data shows how Australia is rebuilding as COVID-19 restrictions ease, 2020. Available: https:// www.abc.net.au/news/2020-05-21/road-to-recovery-from-the-covid19-coronavirus-pandemic/12229514?nw=0 [Accessed 20 Aug 2020].

49 Australian Government Department of Health. Health AGD of coronavirus (COVID-19) common operating picture [online], 2020. Available: https://www.health.gov.au/resources/publications/ coronavirus-covid-19-common-operating-picture [Accessed 24 Sep 2020].

50 Liang M, Gao L, Cheng C, et al. Efficacy of face mask in preventing respiratory virus transmission: a systematic review and metaanalysis. Travel Med Infect Dis 2020;36:101751.

51 Leung NHL, Chu DKW, Shiu EYC, et al. Respiratory virus shedding in exhaled breath and efficacy of face masks. Nat Med 2020;26:676-80.

52 Doung-Ngern P, Suphanchaimat R, Panjangampatthana A, et al. Case-Control study of use of personal protective measures and risk for SARS-CoV 2 infection, Thailand. Emerg Infect Dis 2020;26:2607-16.

53 IHME. COVID-19: What's New for June 252020.

54 The Guardian. NSW makes face masks mandatory as state records seven new Covid cases [online], 2021. Available: http:// www.theguardian.com/australia-news/2021/jan/02/nsw-makesface-masks-mandatory-as-state-records-seven-new-covid-cases [Accessed 25 Jan 2021].

55 The Sydney Morning Herald. Half of Australian population could get COVID vaccine by middle of the year [online], 2021. Available: https://www.smh.com.au/politics/federal/half-of-australianpopulation-could-get-covid-vaccine-by-mid-year-20210107-p56sg8. html [Accessed 25 Jan 2021]

56 WHO. Pandemic fatigue: reinvigorating the public to prevent COVID-19 [online]. Available: https://apps.who.int/iris/bitstream/ handle/10665/335820/WHO-EURO-2020-1160-40906-55390-eng. pdf

57 Kissler SM, Tedijanto C, Goldstein E, et al. Projecting the transmission dynamics of SARS-CoV-2 through the postpandemic period. Science 2020;368:860-8.

58 So AD, Woo J. Reserving coronavirus disease 2019 vaccines for global access: cross sectional analysis. BMJ 2020;371:m4750.

59 The Lancet Microbe. COVID-19 vaccines: the pandemic will not end overnight. Lancet Microbe 2021;2:e1.

60 Maier BF, Brockmann D. Effective containment explains subexponential growth in recent confirmed COVID-19 cases in China. Science 2020;368:742-6.

61 Lee A, Thornley S, Morris AJ, et al. Should countries aim for elimination in the covid-19 pandemic? BMJ 2020;370:m3410. doi:10.1136/bmj.m3410

62 Considerations for implementing and adjusting public health and social measures in the context of COVID-19 [online]. Available: https://www.who.int/publications-detail-redirect/considerationsin-adjusting-public-health-and-social-measures-in-the-context-ofcovid-19-interim-guidance [Accessed 25 Jan 2021]. 